\title{
Hopf Extension Theorem of Measure
}

\author{
Noboru Endou \\ Gifu National College of Technology \\ Japan
}

Yasunari Shidama

Shinshu University

Nagano, Japan

\author{
Hiroyuki Okazaki \\ Shinshu University \\ Nagano, Japan
}

Summary. The authors have presented some articles about Lebesgue type integration theory. In our previous articles [12, 13, 26], we assumed that some $\sigma$-additive measure existed and that a function was measurable on that measure. However the existence of such a measure is not trivial. In general, because the construction of a finite additive measure is comparatively easy, to induce a $\sigma$ additive measure a finite additive measure is used. This is known as an E. Hopf's extension theorem of measure [15].

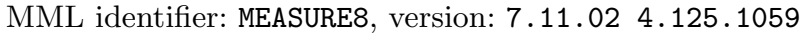

The articles [11], [23], [1], [24], [22], [8], [25], [10], [9], [2], [20], [26], [6], [5], [7], [13], [4], [12], [3], [16], [19], [18], [27], [21], [17], and [14] provide the notation and terminology for this paper.

\section{The Outer Measure Induced by the Finite Additive Measure}

For simplicity, we follow the rules: $X$ denotes a set, $F$ denotes a field of subsets of $X, M$ denotes a measure on $F, A, B$ denote subsets of $X, S_{1}$ denotes a sequence of subsets of $X, s_{1}, s_{2}, s_{3}$ denote sequences of extended reals, and $n, k$ denote natural numbers.

We now state three propositions:

(1) Ser $s_{1}=\left(\sum_{\alpha=0}^{\kappa}\left(s_{1}\right)(\alpha)\right)_{\kappa \in \mathbb{N}}$.

$(2)^{1} \quad$ If $s_{1}$ is non-negative, then $s_{1}$ is summable and $\sum s_{1}=\sum s_{1}$.

\footnotetext{
${ }^{1}$ The translation of Mizar functor SUM introduced in [4] was changed from $\sum$ to $\bar{\sum}$.
} 
(3) Suppose $s_{2}$ is non-negative and $s_{3}$ is non-negative and for every natural number $n$ holds $s_{1}(n)=s_{2}(n)+s_{3}(n)$. Then $s_{1}$ is non-negative and $\sum s_{1}=$ $\bar{\sum} s_{2}+\sum s_{3}$ and $\sum s_{1}=\sum s_{2}+\sum s_{3}$.

Let us consider $X, F$. One can check that there exists a function from $\mathbb{N}$ into $F$ which is disjoint valued.

Let us consider $X, F$. A finite sequence of elements of $2^{X}$ is said to be a finite sequence of elements of $F$ if:

(Def. 1) For every natural number $k$ such that $k \in \operatorname{dom}$ it holds $\operatorname{it}(k) \in F$.

Let us consider $X, F$. Observe that there exists a finite sequence of elements of $F$ which is disjoint valued.

Let us consider $X, F$. A disjoint valued finite set sequence of $F$ is a disjoint valued finite sequence of elements of $F$.

Let us consider $X, F$. A sequence of separated subsets of $F$ is a disjoint valued function from $\mathbb{N}$ into $F$.

Let us consider $X, F$. A sequence of subsets of $X$ is said to be a set sequence of $F$ if:

(Def. 2) For every natural number $n$ holds it $(n) \in F$.

Let us consider $X, A, F$. A set sequence of $F$ is said to be a covering of $A$ in $F$ if:

(Def. 3) $A \subseteq \bigcup$ rng it.

In the sequel $F_{1}$ denotes a set sequence of $F$ and $C_{1}$ denotes a covering of $A$ in $F$.

Let us consider $X, F, F_{1}, n$. Then $F_{1}(n)$ is an element of $F$.

Let us consider $X, F, S_{1}$. A function from $\mathbb{N}$ into $\left(2^{X}\right)^{\mathbb{N}}$ is said to be a covering of $S_{1}$ in $F$ if:

(Def. 4) For every element $n$ of $\mathbb{N}$ holds it $(n)$ is a covering of $S_{1}(n)$ in $F$.

In the sequel $C_{2}$ is a covering of $S_{1}$ in $F$.

Let us consider $X, F, M, F_{1}$. The functor $\operatorname{vol}\left(M, F_{1}\right)$ yielding a sequence of extended reals is defined as follows:

(Def. 5) For every $n$ holds $\left(\operatorname{vol}\left(M, F_{1}\right)\right)(n)=M\left(F_{1}(n)\right)$.

One can prove the following proposition

(4) $\operatorname{vol}\left(M, F_{1}\right)$ is non-negative.

Let us consider $X, F, S_{1}, C_{2}$ and let $n$ be an element of $\mathbb{N}$. Then $C_{2}(n)$ is a covering of $S_{1}(n)$ in $F$.

Let us consider $X, F, S_{1}, M, C_{2}$. The functor $\operatorname{Volume}\left(M, C_{2}\right)$ yielding a sequence of extended reals is defined as follows:

(Def. 6) For every element $n$ of $\mathbb{N}$ holds $\left(\operatorname{Volume}\left(M, C_{2}\right)\right)(n)=\bar{\sum} \operatorname{vol}\left(M, C_{2}(n)\right)$.

The following proposition is true

(5) $0 \leq\left(\operatorname{Volume}\left(M, C_{2}\right)\right)(n)$. 
Let us consider $X, F, M, A$. The functor $\operatorname{Svc}(M, A)$ yielding a subset of $\overline{\mathbb{R}}$ is defined as follows:

(Def. 7) For every extended real number $x$ holds $x \in \operatorname{Svc}(M, A)$ iff there exists a covering $C_{1}$ of $A$ in $F$ such that $x=\bar{\sum} \operatorname{vol}\left(M, C_{1}\right)$.

Let us consider $X, A, F, M$. Observe that $\operatorname{Svc}(M, A)$ is non empty.

Let us consider $X, F, M$. The Caratheodory measure determined by $M$ is a function from $2^{X}$ into $\overline{\mathbb{R}}$ and is defined by:

(Def. 8) For every subset $A$ of $X$ holds (the Caratheodory measure determined by $M)(A)=\inf \operatorname{Svc}(M, A)$.

The function InvPairFunc from $\mathbb{N}$ into $\mathbb{N} \times \mathbb{N}$ is defined by:

(Def. 9) $\quad$ InvPairFunc $=$ PairFunc $^{-1}$.

Let us consider $X, F, S_{1}, C_{2}$. The functor On $C_{2}$ yielding a covering of $\cup \operatorname{rng} S_{1}$ in $F$ is defined by:

(Def. 10) For every natural number $n$ holds $\left(\right.$ On $\left.C_{2}\right)(n)=$ $C_{2}(\operatorname{pr} 1(\operatorname{InvPairFunc})(n))(\operatorname{pr} 2(\operatorname{InvPairFunc})(n))$.

The following propositions are true:

(6) Let $k$ be an element of $\mathbb{N}$. Then there exists a natural number $m$ such that for every sequence $S_{1}$ of subsets of $X$ and for every covering $C_{2}$ of $S_{1}$ in $F$ holds $\left(\sum_{\alpha=0}^{\kappa}\left(\operatorname{vol}\left(M, \text { On } C_{2}\right)\right)(\alpha)\right)_{\kappa \in \mathbb{N}}(k) \leq$ $\left(\sum_{\alpha=0}^{\kappa}\left(\operatorname{Volume}\left(M, C_{2}\right)\right)(\alpha)\right)_{\kappa \in \mathbb{N}}(m)$.

(7) $\inf \operatorname{Svc}\left(M, \bigcup \operatorname{rng} S_{1}\right) \leq \bar{\sum} \operatorname{Volume}\left(M, C_{2}\right)$.

(8) If $A \in F$, then $A, \emptyset_{X}$ followed by $\emptyset_{X}$ is a covering of $A$ in $F$.

(9) Let $X$ be a set, $F$ be a field of subsets of $X, M$ be a measure on $F$, and $A$ be a set. If $A \in F$, then (the Caratheodory measure determined by $M)(A) \leq M(A)$.

(10) The Caratheodory measure determined by $M$ is non-negative.

(11) (The Caratheodory measure determined by $M)(\emptyset)=0$.

(12) If $A \subseteq B$, then (the Caratheodory measure determined by $M)(A) \leq$ (the Caratheodory measure determined by $M)(B)$.

(13) (The Caratheodory measure determined by $M)\left(\bigcup \operatorname{rng} S_{1}\right) \leq \bar{\sum}($ (the Caratheodory measure determined by $M) \cdot S_{1}$ ).

(14) The Caratheodory measure determined by $M$ is a Caratheodor's measure on $X$.

Let $X$ be a set, let $F$ be a field of subsets of $X$, and let $M$ be a measure on $F$. Then the Caratheodory measure determined by $M$ is a Caratheodor's measure on $X$. 


\section{Hopf Extension Theorem}

Let $X$ be a set, let $F$ be a field of subsets of $X$, and let $M$ be a measure on $F$. We say that $M$ is completely-additive if and only if:

(Def. 11) For every sequence $F_{1}$ of separated subsets of $F$ such that $\bigcup \operatorname{rng} F_{1} \in F$ holds $\bar{\sum}\left(M \cdot F_{1}\right)=M\left(\bigcup \operatorname{rng} F_{1}\right)$.

The following propositions are true:

(15) The partial unions of $F_{1}$ are a set sequence of $F$.

(16) The partial diff-unions of $F_{1}$ are a set sequence of $F$.

(17) Suppose $A \in F$. Then there exists a sequence $F_{1}$ of separated subsets of $F$ such that $A=\bigcup \operatorname{rng} F_{1}$ and for every natural number $n$ holds $F_{1}(n) \subseteq$ $C_{1}(n)$.

(18) Suppose $M$ is completely-additive. Let $A$ be a set. If $A \in F$, then $M(A)=$ (the Caratheodory measure determined by $M)(A)$.

In the sequel $C$ is a Caratheodor's measure on $X$.

We now state three propositions:

(19) If for every subset $B$ of $X$ holds $C(B \cap A)+C(B \cap(X \backslash A)) \leq C(B)$, then $A \in \sigma$-Field $(C)$.

(20) $F \subseteq \sigma$-Field(the Caratheodory measure determined by $M$ ).

(21) Let $X$ be a set, $F$ be a field of subsets of $X, F_{1}$ be a set sequence of $F$, and $M$ be a function from $F$ into $\overline{\mathbb{R}}$. Then $M \cdot F_{1}$ is a sequence of extended reals.

Let $X$ be a set, let $F$ be a field of subsets of $X$, let $F_{1}$ be a set sequence of $F$, and let $g$ be a function from $F$ into $\overline{\mathbb{R}}$. Then $g \cdot F_{1}$ is a sequence of extended reals.

One can prove the following proposition

(22) Let $X$ be a set, $S$ be a $\sigma$-field of subsets of $X, S_{2}$ be a sequence of subsets of $S$, and $M$ be a function from $S$ into $\overline{\mathbb{R}}$. Then $M \cdot S_{2}$ is a sequence of extended reals.

Let $X$ be a set, let $S$ be a $\sigma$-field of subsets of $X$, let $S_{2}$ be a sequence of subsets of $S$, and let $g$ be a function from $S$ into $\overline{\mathbb{R}}$. Then $g \cdot S_{2}$ is a sequence of extended reals.

Next we state several propositions:

(23) Let $F, G$ be functions from $\mathbb{N}$ into $\overline{\mathbb{R}}$ and $n$ be a natural number. Suppose that for every natural number $m$ such that $m \leq n$ holds $F(m) \leq G(m)$. Then $(\operatorname{Ser} F)(n) \leq(\operatorname{Ser} G)(n)$.

(24) For all $X, C$ and for every sequence $s_{1}$ of separated subsets of $\sigma$-Field $(C)$ holds $\bigcup \operatorname{rng} s_{1} \in \sigma$-Field $(C)$ and $C\left(\bigcup \operatorname{rng} s_{1}\right)=\sum\left(C \cdot s_{1}\right)$.

(25) For all $X, C$ and for every sequence $s_{1}$ of subsets of $\sigma$-Field $(C)$ holds $\bigcup s_{1} \in \sigma-\operatorname{Field}(C)$. 
(26) Let $X$ be a non empty set, $S$ be a $\sigma$-field of subsets of $X, M$ be a $\sigma$ measure on $S$, and $S_{2}$ be a sequence of subsets of $S$. If $S_{2}$ is non-decreasing, then $\lim \left(M \cdot S_{2}\right)=M\left(\lim S_{2}\right)$.

(27) If $F_{1}$ is non-decreasing, then $M \cdot F_{1}$ is non-decreasing.

(28) If $F_{1}$ is descending, then $M \cdot F_{1}$ is non-increasing.

(29) Let $X$ be a set, $S$ be a $\sigma$-field of subsets of $X, M$ be a $\sigma$-measure on $S$, and $S_{2}$ be a sequence of subsets of $S$. If $S_{2}$ is non-decreasing, then $M \cdot S_{2}$ is non-decreasing.

(30) Let $X$ be a set, $S$ be a $\sigma$-field of subsets of $X, M$ be a $\sigma$-measure on $S$, and $S_{2}$ be a sequence of subsets of $S$. If $S_{2}$ is descending, then $M \cdot S_{2}$ is non-increasing.

(31) Let $X$ be a non empty set, $S$ be a $\sigma$-field of subsets of $X, M$ be a $\sigma$ measure on $S$, and $S_{2}$ be a sequence of subsets of $S$. If $S_{2}$ is descending and $M\left(S_{2}(0)\right)<+\infty$, then $\lim \left(M \cdot S_{2}\right)=M\left(\lim S_{2}\right)$.

Let $X$ be a set, let $F$ be a field of subsets of $X$, let $S$ be a $\sigma$-field of subsets of $X$, let $m$ be a measure on $F$, and let $M$ be a $\sigma$-measure on $S$. We say that $M$ is an extension of $m$ if and only if:

(Def. 12) For every set $A$ such that $A \in F$ holds $M(A)=m(A)$.

The following four propositions are true:

(32) Let $X$ be a non empty set, $F$ be a field of subsets of $X$, and $m$ be a measure on $F$. If there exists a $\sigma$-measure on $\sigma(F)$ which is an extension of $m$, then $m$ is completely-additive.

(33) Let $X$ be a non empty set, $F$ be a field of subsets of $X$, and $m$ be a measure on $F$. Suppose $m$ is completely-additive. Then there exists a $\sigma$-measure $M$ on $\sigma(F)$ such that $M$ is an extension of $m$ and $M=$ $\sigma$-Meas(the Caratheodory measure determined by $m)\lceil\sigma(F)$.

(34) If for every $n$ holds $M\left(F_{1}(n)\right)<+\infty$, then $M($ (the partial unions of $\left.\left.F_{1}\right)(k)\right)<+\infty$.

(35) Let $X$ be a non empty set, $F$ be a field of subsets of $X$, and $m$ be a measure on $F$. Suppose that

(i) $m$ is completely-additive, and

(ii) there exists a set sequence $A_{1}$ of $F$ such that for every natural number $n$ holds $m\left(A_{1}(n)\right)<+\infty$ and $X=\bigcup \operatorname{rng} A_{1}$.

Let $M$ be a $\sigma$-measure on $\sigma(F)$. Suppose $M$ is an extension of $m$. Then $M=\sigma$-Meas(the Caratheodory measure determined by $m)\lceil\sigma(F)$.

\section{REFERENCES}

[1] Grzegorz Bancerek. The ordinal numbers. Formalized Mathematics, 1(1):91-96, 1990.

[2] Grzegorz Bancerek and Krzysztof Hryniewiecki. Segments of natural numbers and finite sequences. Formalized Mathematics, 1(1):107-114, 1990. 
[3] Józef Białas. Infimum and supremum of the set of real numbers. Measure theory. Formalized Mathematics, 2(1):163-171, 1991.

[4] Józef Białas. Series of positive real numbers. Measure theory. Formalized Mathematics, 2(1):173-183, 1991.

[5] Józef Białas. Several properties of the $\sigma$-additive measure. Formalized Mathematics, 2(4):493-497, 1991.

[6] Józef Białas. The $\sigma$-additive measure theory. Formalized Mathematics, 2(2):263-270, 1991.

[7] Józef Białas. Properties of Caratheodor's measure. Formalized Mathematics, 3(1):67-70, 1992.

[8] Czesław Byliński. Functions and their basic properties. Formalized Mathematics, 1(1):5565, 1990.

[9] Czesław Byliński. Functions from a set to a set. Formalized Mathematics, 1(1):153-164, 1990.

[10] Czesław Byliński. Partial functions. Formalized Mathematics, 1(2):357-367, 1990.

[11] Czesław Byliński. Some basic properties of sets. Formalized Mathematics, 1(1):47-53, 1990.

[12] Noboru Endou, Keiko Narita, and Yasunari Shidama. The Lebesgue monotone convergence theorem. Formalized Mathematics, 16(2):167-175, 2008, doi:10.2478/v10037-0080023-1.

[13] Noboru Endou and Yasunari Shidama. Integral of measurable function. Formalized Mathematics, 14(2):53-70, 2006, doi:10.2478/v10037-006-0008-x.

[14] Adam Grabowski. On the Kuratowski limit operators. Formalized Mathematics, 11(4):399-409, 2003.

[15] P. R. Halmos. Measure Theory. Springer-Verlag, 1987.

[16] Krzysztof Hryniewiecki. Recursive definitions. Formalized Mathematics, 1(2):321-328, 1990.

[17] Franz Merkl. Dynkin's lemma in measure theory. Formalized Mathematics, 9(3):591-595, 2001.

[18] Andrzej Nędzusiak. Probability. Formalized Mathematics, 1(4):745-749, 1990.

[19] Andrzej Nędzusiak. $\sigma$-fields and probability. Formalized Mathematics, 1(2):401-407, 1990.

[20] Beata Padlewska. Families of sets. Formalized Mathematics, 1(1):147-152, 1990.

[21] Karol Pąk. The Nagata-Smirnov theorem. Part II. Formalized Mathematics, 12(3):385$389,2004$.

[22] Andrzej Trybulec. Binary operations applied to functions. Formalized Mathematics, 1(2):329-334, 1990.

[23] Zinaida Trybulec. Properties of subsets. Formalized Mathematics, 1(1):67-71, 1990.

[24] Edmund Woronowicz. Relations and their basic properties. Formalized Mathematics, 1(1):73-83, 1990.

[25] Edmund Woronowicz. Relations defined on sets. Formalized Mathematics, 1(1):181-186,

[26] Hiroshi Yamazaki, Noboru Endou, Yasunari Shidama, and Hiroyuki Okazaki. Inferior limit, superior limit and convergence of sequences of extended real numbers. Formalized Mathematics, 15(4):231-236, 2007, doi:10.2478/v10037-007-0026-3.

[27] Bo Zhang, Hiroshi Yamazaki, and Yatsuka Nakamura. Set sequences and monotone class. Formalized Mathematics, 13(4):435-441, 2005. 